Nguyen X.-C.

Nagoya Math. J.

Vol. 103 (1986), 15-27

\title{
ON THE POWER SERIES REPRESENTATION OF SMOOTH CONFORMAL MARTINGALES
}

\author{
NGUYEN XUAN-LOC
}

We introduce here the notion of (stochastically) differentiable process with respect to a fixed conformal martingale and compute the remainder term of the Taylor expansion of the given process (Definition 1 and Proposition 3). An a-priori estimate in the $L^{2}$-norm of the above mentioned remainder term is given and consequently a power series representation of smooth conformal martingales is obtained (Theorem 4).

The results in this note on the one hand extend those of our previous works on smooth martingales with respect to the stopped complex Brownian motion (see [3]) and on the other hand can be applied to approximate the solutions of complex Ito-diffusion equations (see Applications). Furthermore they give a positive answer to an open question raised by M. Yor (see [5]).

Power Series representation of smooth conformal martingales with respect to a fixed martingale

For the convenience of the reader let us recall briefly the notion of conformal martingales and their associated Ito formula. We refer to the original work of Getoor and Sharpe for the complete literature of the theory of conformal martingales (see [2]). By probability system we always mean a collection $\Sigma=\left(\Omega, \mathscr{F},\left(\mathscr{F}_{t}\right)_{t \geq 0}, P\right)$ which consists of a probability space $(\Omega, \mathscr{F}, P)$ and an increasing family $\left(\mathscr{F}_{t}\right)_{t \geq 0}$ of sub $\sigma$-fields of $\mathscr{F}$ with the following standard hypotheses:

$$
\begin{aligned}
& -\left(\mathscr{F}_{t}\right)_{t \geq 0} \text { is right-continuous, } \\
& -\mathscr{F}_{0} \text { contains all null sets of } \mathscr{F} .
\end{aligned}
$$

We always denote by $\mathscr{M}_{c}$ the class of continuous complex local martingales for $\Sigma$. Thus $W \in \mathscr{M}_{c}$ iff its real and imaginary parts are continuous

Received July 11, 1983.

Revised December 15, 1984. 
local martingales. In other words,

$W=W_{0}+U+i V$ where $W_{0}$ is an $\mathscr{F}_{0}$-measurable complex random

(1) variable and where $U, V$ belong to the class $\mathscr{L}_{c}$ of continuous, real local martingales which are null at $t=0$.

Let $\mathscr{A}_{c}$ be the class of continuous, real processes for $\Sigma$ which are null at $t=0$ and which are locally of integrable total variations.

For $W_{1}, W_{2} \in \mathscr{M}_{c}$ the process $\left\langle W_{1}, W_{2}\right\rangle$, defined by complex polarization, is the unique member of $\mathscr{A}_{c}+i \mathscr{A}_{c}$ such that

$$
W_{1} \cdot \bar{W}_{2}-\left\langle W_{1}, W_{2}\right\rangle \text { is an element of } \mathscr{M}_{c} \text {. }
$$

One finds that $\left\langle W_{1}, W_{2}\right\rangle=\left\langle W_{1}-W_{1}(0), W_{2}-W_{2}(0)\right\rangle$ and that $\left\langle W_{1}, W_{2}\right\rangle$ is linear in $W_{1}$, conjugate linear in $W_{2}$. It follows at once from (2) that for an element $W=W_{0}+U+i V \in \mathscr{M}_{c}$ we have:

$$
\left\{\begin{array}{l}
\langle W, W\rangle=\langle U, U\rangle+\langle V, V\rangle \text { and, } \\
\langle W, \bar{W}\rangle=\langle U, U\rangle-\langle V, V\rangle+2 i\langle U, V\rangle
\end{array}\right.
$$

In the following we denote also by $\langle W\rangle$ (resp. $\langle U\rangle$ ) the process $\langle W, W\rangle$ (resp. $\langle U, U\rangle$ ).

Definition A (see [2], (5.1)). An element $W=W_{0}+U+i V$ of $\mathscr{M}_{c}$ is called conformal provided that:

$$
\langle U, U\rangle=\langle V, V\rangle \text { and }\langle U, V\rangle=0 .
$$

The class of conformal martingales for the probability system $\Sigma$ will be denoted by $\mathscr{C}$. Clearly $W \in \mathscr{C}$ iff $\bar{W} \in \mathscr{C}$ and the stopped process $W^{T}=$ $\left(W_{T \wedge t}\right)$ also belongs to $\mathscr{C}$ for every $\left(\mathscr{F}_{t}\right)$-stopping time $T$.

Now fix an element $W=W_{0}+U+i V \in \mathscr{M}_{c}$ and let $L_{\mathrm{loc}}^{2}(W)$ be the class of previsible complex processes $C=\left(C_{s}\right)$ such that $\left(\int_{0}^{t}\left|C_{s}\right|^{2} d\langle W\rangle_{s}\right)$ is locally integrable. If $W$ is furthermore conformal then:

$$
L_{\mathrm{loc}}^{2}(W)=L_{\mathrm{loc}}^{2}(U)+i L_{\mathrm{loc}}^{2}(U)
$$

(notice $L_{\mathrm{loc}}^{2}(U)=L_{\mathrm{loc}}^{2}(V)$ ).

To save spaces let us denote in the rest of the paper by $C * W$ the stochastic integral of an element of $C=\left(C_{s}\right) \in L_{\text {loc }}^{2}(W)$ with respect to $(W-W(0))$ and write: 


$$
\begin{aligned}
& C * W(t)=\int_{0}^{t} C_{s} \circ d W_{s} \\
& \left.\quad \text { (instead of } C \cdot(W-W(0))(t)=\int_{0}^{t} C_{s} \cdot d(W-W(0))_{s}\right) .
\end{aligned}
$$

The notation $C * W(K \in N)$ will be used to denote the iterated stochastic integral:

(6') $\quad C * W(t)=C * W * \cdots * W(t)$ (the operation $*$ is repeated $K$ times).

Notice that if $C \in L_{\mathrm{loc}}^{2}(W)$ then by the continuity of the paths $C^{K} * W$ also belongs to $L_{\mathrm{loc}}^{2}(W)$ and furthermore it is not difficult to see that

$$
\int_{0}^{t}\left|C{ }^{K} W_{s}\right|^{2} d\langle W\rangle_{s}=\int_{0}^{t}\left(\int_{0}^{s}\left|C^{K-1} W_{u}\right|^{2} d\langle W\rangle_{u}\right) \cdot d\langle W\rangle_{s}+M_{t} \cdot\langle W\rangle_{t}
$$

where $\left(M_{t}\right)$ is the local martingale given by

$$
M_{t} \equiv\left|C_{*}^{K-1} W_{t}\right|^{2}-\left\langle C_{*}^{K-1} W\right\rangle_{t} .
$$

The following Ito-formula for the class $\mathscr{C}$ of conformal martingales of Getoor and Sharpe will be used frequently in the sequel.

Theorem B (see [2], (5.4)). Let $D$ be a domain in $C^{n}$ and let $Z^{1}, \ldots$, $Z^{n} \in \mathscr{C}$ such that

$$
P\left(\left(Z^{1}, \cdots, Z^{n}\right) \in D \text { for any } t \geq 0\right)=1 .
$$

If $F$ is holomorphic in a neighbourhood of $D$, then

$$
\begin{aligned}
F\left(Z_{t}^{1}, \cdots, Z_{t}^{n}\right) & =F\left(Z_{0}^{1}, \cdots, Z_{0}^{n}\right)+\sum_{j=1}^{n} \int_{0}^{t} \frac{\partial F}{\partial z_{j}}\left(Z_{s}^{1}, \cdots, Z_{s}^{n}\right) \cdot d Z_{s}^{j} \\
& +\frac{1}{2} \sum_{j, k=1}^{n} \int_{0}^{t} \frac{\partial^{2} F}{\partial z_{j} \partial z_{k}}\left(Z_{s}^{1}, \cdots, Z_{s}^{n}\right) \cdot d\left\langle Z^{j}, \bar{Z}^{k}\right\rangle_{s}
\end{aligned}
$$

(Ito formula).

Consequently if $Z^{1}, \cdots, Z^{n} \in \mathscr{C}$ and $\left\langle Z^{j}, \bar{Z}^{k}\right\rangle=0$ for all $j, k$ (notice $\left\langle Z^{j}, \bar{Z}^{j}\right\rangle$ $=0$ automatically) then under the same hypothesis on $F$, one has

$$
F\left(Z_{t}^{1}, \cdots, Z_{t}^{n}\right)=F\left(Z_{0}^{1}, \cdots, Z_{0}^{n}\right)+\sum_{j=1}^{n} \int_{0}^{t} \frac{\partial F}{\partial z_{j}}\left(Z_{s}^{1}, \cdots, Z_{s}^{n}\right) \cdot d Z_{s}^{j},
$$

i.e., $F\left(Z_{t}^{1}, \cdots, Z_{t}^{n}\right)$ belongs again to $\mathscr{C}$.

Definition 1 . a) Let $W$ be a fixed element of $\mathscr{C}$. A conformal martingale $\phi=(\phi(s))$ of $\mathscr{C}$ is said to be $n$-times differentiable with respect 
to $W$ (abbrev. $n$-times $W$-differentiable) provided that there are $n$ elements $\phi^{(i)} \in L_{\mathrm{loc}}^{2}(W)(1 \leq i \leq n)$ such that:

$$
\left\{\begin{array}{l}
\phi-\phi(0)=\phi^{1} * W \\
\phi^{(i)}-\phi^{(i)}(0)=\phi^{(i+1)} * W \quad(1 \leq i \leq n-1) .
\end{array}\right.
$$

b) A conformal martingale $\phi \in \mathscr{C}$ is said to be smooth with respect to $W$ if it is $W$-differentiable infinitely many times.

Remarks. 1) It is implicitly stated in the formula (8) of the Definition 1 that $\phi^{(i)} \in \mathscr{C}$ for $i=1,2, \cdots, n-1$.

2) The particular case, where $W=\left(B_{T \wedge t}\right)$ is chosen to be the complex Brownian motion stopped at the stopping time $T$, was introduced in an earlier work [3] and a smooth martingale (with respect to $W=\left(B_{T \wedge t}\right)$ ) is called in [3] a locally analytic processes.

LEMMA 2. a) Let $W$ be a fixed conformal martingale and let $n$ be an integer then the process:

$$
W^{n}=(W(t)-W(0))^{n}
$$

belongs to $\mathscr{C}$ and furthermore, for every integer $1 \leq k \leq n$ we have

$$
W^{n}=n(n-1) \cdots(n-k)\left(W^{(n-k)}\right)^{k} * W
$$

(see (6) and $\left(6^{\prime}\right)$ for the definition of $* W$ ).

b) Let $\phi=(\phi(t))$ be a $(n+1)$ times $W$-differentiable martingale then $\phi$ can be written as follows:

$$
\phi(t)=\phi(0)+\sum_{i=1}^{n} \frac{\phi^{(i)}(0)}{i !} W^{i}(t)+\left(\phi_{*}^{(n+1)} \stackrel{(n+1)}{*} W\right)(t) .
$$

Proof. (a) Since $\langle W, \bar{W}\rangle=0$, it follows from Ito-formula (7) applying to $f(z)=z^{n}$ that:

$$
W^{n}(t)=n W^{n-1} * W(t) .
$$

From the above equality (10) can be obtained easily by iteration.

b) Suppose first that $n=0$, then (11) says nothing else than the definition of $W$-differentiable martingale, i.e.,

$$
\phi(t)=\phi(0)+\int_{0}^{t} \phi^{(1)}(s) \cdot d W_{s} .
$$

Now suppose that (11) is true for $n=k$ and that $\phi$ is a $(k+1)$-times $W$ - 
differentiable martingale then we have:

$$
\phi(t)=\phi(0)+\sum_{i=1}^{k-1} \frac{\phi^{(i)}(0)}{i !} \cdot W^{i}(t)+\left(\phi^{(k)} \stackrel{*}{*} W\right)(t) .
$$

On the other hand since $\phi^{(k)}$ can be written as follows:

$$
\phi^{(k)}(s)=\phi^{(k)}(0)+\left(\phi^{(k+1)} * W\right)(s),
$$

thus the last term of the right hand side member of (12) turns out to be:

$$
\begin{aligned}
\left(\phi^{(k)}{ }^{k} * W\right)(t) & =\left(\phi^{(k)}(0)+\phi^{(k+1)} * W\right)^{k} * W(t) \\
& =\phi^{(k)}(0)^{k} * W(t)+\phi^{(k+1)}{ }_{*}^{(k+1)} W(t) \\
& =\frac{\phi^{(k)}(0)}{k !} \cdot W^{k}(t)+\phi^{(k+1)}{ }_{*}^{(k+1)} W(t)
\end{aligned}
$$

by (10). Hence (11) is also true for $n=k+1$.

q.e.d.

Remark. Notice that $\phi^{(k)}(0)(0 \leq k \leq n)$ is $\mathscr{F}_{0}$-measurable hence they behave like a constant in the stochastic integration procedure and that if $\psi \in L_{\mathrm{loc}}^{2}(W)$ then so is $\psi * W$.

Let $W \in \mathscr{C}$ be a fixed conformal martingale and let $\langle W\rangle=\left(\langle W\rangle_{s}\right)$ be the continuous increasing process associated with $W$. For $t \geq 0$ let us define the stopping-time $\tau(t)$ as follows:

$$
\tau(t, \omega)=\left\{\begin{array}{l}
\inf \left\{s>0:\langle W\rangle_{s}(\omega) \geq t\right\} \\
+\infty \text { if the set of } s \text { such that }\langle W\rangle_{s}(\omega) \geq t \text { is empty } .
\end{array}\right.
$$

Proposition 3. a) Let $\phi=(\phi(t, \cdot))$ be $a(n+1)$-times $W$-differentiable conformal martingale with derivatives $\phi^{(i)}(1 \leq i \leq n+1)$. Suppose furthermore that $E\left(|\phi(\tau(t, \cdot))|^{2}\right)<+\infty$, for some fixed $t>0$, then for every $t_{0}<t$ :

$$
\begin{aligned}
E\left(|\phi(\tau(t, \cdot))|^{2}\right)= & E\left(\left|\phi\left(\tau\left(t_{0}, \cdot\right)\right)\right|^{2}\right)+\int_{t_{0}}^{t} d t_{n} \cdots \int_{t_{0}}^{t_{1}} E\left(|\phi(\tau(s, \cdot))|^{2}\right) d s \\
& +\sum_{i=1}^{n} E\left(\left|\phi^{(i)}(\tau(t, \cdot))\right|^{2}\right) \frac{\left(t-t_{0}\right)^{i}}{i !} \\
& -\sum_{i=1}^{n+1} E\left(\left|\phi^{i}(\infty, \cdot)\right|^{2} \cdot \frac{\left(t-t_{0} \vee\langle W\rangle_{\infty}\right)^{i}}{i !} ;\langle W\rangle_{\infty} \leq t\right)
\end{aligned}
$$

b) Consequently if $\phi$ is a W-smooth conformal martingale such that $E\left(|\phi(\tau(t, \cdot))|^{2}\right)<+\infty$, then the two following conditions are equivalent: 


$$
\begin{gathered}
\left.\sum_{i=1}^{\infty} E\left(\left|\phi^{(i)}(\infty, \cdot)\right|^{2}\right) \cdot \frac{\left(t-\langle W\rangle_{\infty} \vee t_{0}\right)^{i}}{i !} ;\langle W\rangle_{\infty} \leq t\right)<+\infty \\
\sum_{i=1}^{\infty} E\left(\left|\phi^{(i)}\left(\tau\left(t_{0}, \cdot\right)\right)\right|^{2}\right) \cdot \frac{\left(t-t_{0}\right)^{i}}{i !}<+\infty
\end{gathered}
$$

Proof. a) Let $0 \leq t_{0} \leq t$,

$$
\begin{aligned}
& E\left(|\phi(\tau(t, \cdot))|^{2}-\left|\phi\left(\tau\left(t_{0}, \cdot\right)\right)\right|^{2} \mid \mathscr{F}_{\tau\left(t_{0}, \cdot\right)}\right) \\
& \quad=E\left(\int_{\tau\left(t_{0}, \cdot\right)}^{\tau(t, \cdot)}\left|\phi^{1}(s, \cdot)\right|^{2} d\langle W\rangle_{s} \mid \mathscr{F}_{\tau\left(t_{0}, \cdot\right)}\right) \\
& \quad=E\left(\int_{t_{0} \wedge\langle W\rangle_{\infty}}^{t \wedge\langle W\rangle_{\infty}}\left|\phi^{1}(\tau(s, \cdot))\right|^{2} d s \mid \mathscr{F}_{\tau\left(t_{0}, \cdot\right)}\right) .
\end{aligned}
$$

On the other hand since $\{\omega ; \tau(t, \omega)=\infty\}=\left\{\omega ;\langle W\rangle_{\infty} \leq t\right\}$ and since

$$
\int_{t_{0} \wedge\langle W\rangle_{\infty}}^{t \wedge\langle W\rangle_{\infty}}\left|\phi^{1}(\tau(s, \cdot))\right|^{2} d s= \begin{cases}\int_{t_{0}}^{t}\left|\phi^{1}(\tau(s, \cdot))\right|^{2} d s & \text { on } t<\langle W\rangle_{\infty} \\ \int_{t_{0}}^{\langle W\rangle_{\infty}}\left|\phi^{1}(\tau(s, \cdot))\right|^{2} d s & \text { on } t_{0}<\langle W\rangle_{\infty} \leq t, \\ 0 & \text { on }\langle W\rangle_{\infty} \leq t_{0}\end{cases}
$$

we have, by iterating on $E\left(\left|\phi^{1}(\tau(s, \cdot))\right|^{2}\right)$,

$$
\begin{aligned}
E\left(|\phi(\tau(t, \cdot))|^{2}\right)-E\left(\left|\phi\left(\tau\left(t_{0}, \cdot\right)\right)\right|^{2}\right) \\
=E\left(\int_{t_{0}}^{t}\left|\phi^{1}(\tau(s, \cdot))\right|^{2} d s ; t<\langle W\rangle_{\infty}\right) \\
\quad+E\left(\int_{t_{0}}^{\langle W\rangle_{\infty}}\left|\phi^{1}(\tau(s, \cdot))\right|^{2} d s ; t_{0}<\langle W\rangle_{\infty} \leq t\right) \\
=\int_{t_{0}}^{t} E\left(\left|\phi^{1}(\tau(s, \cdot))\right|^{2}\right) d s-E\left(\left|\phi^{1}(\infty, \cdot)\right|^{2}\left(t-t_{0} \vee\langle W\rangle_{\infty}\right) ;\langle W\rangle_{\infty} \leq t\right) \\
=E\left(\left|\phi^{1}\left(\tau\left(t_{0}, \cdot\right)\right)\right|^{2}\right)\left(t-t_{0}\right)+\int_{t_{0}}^{t} d t_{n-1} \int_{t_{0}}^{t_{n-1}} E\left(\left|\phi^{2}\left(t_{n}, \cdot\right)\right|^{2}\right) d t_{n} \\
\quad-E\left(\left|\phi^{1}(\infty, \cdot)\right|^{2}\left(t-t_{0} \vee\langle W\rangle_{\infty}\right) ;\langle W\rangle_{\infty} \leq t\right) \\
\quad-E\left(\int_{t_{0}}^{t} d t_{n}\left|\phi^{2}(\infty, \cdot)\right|^{2} d s\left(t_{n}-t_{0} \vee\langle W\rangle_{\infty}\right) ;\langle W\rangle_{\infty} \leq t_{n}\right) .
\end{aligned}
$$

The last term of the right-hand side is:

$$
\begin{gathered}
\int_{t_{0}}^{t} d t_{n} E\left(\left|\phi^{2}(\infty, \cdot)\right|^{2}\left(t_{n}-t_{0} \vee\langle W\rangle_{\infty}\right) ;\langle W\rangle_{\infty} \leq t_{n}\right) \\
=E\left(\left|\phi^{2}(\infty, \cdot)\right|^{2} \frac{\left(t-t_{0} \bigvee\langle W\rangle_{\infty}\right)^{2}}{2} ;\langle W\rangle_{\infty} \leq t\right) .
\end{gathered}
$$

Thus by iteration again on $E\left(\left|\phi^{2}\left(\tau\left(t_{n}, \cdot\right)\right)\right|^{2}\right)$ and so on we get finally (13). 
b) Suppose that (14) (resp. (15)) is true, by letting $n$ to $+\infty$ in (13) we get (15) (resp. (14)).

THEOREM 4. Let $\phi=(\phi(t, \cdot))$ be a W-smooth conformal martingale such that for every couple reals $t>t_{0} \geq 0$ :

$$
\begin{aligned}
& E\left(|\phi(\tau(t, \cdot))|^{2}\right)<\infty \quad \text { and, } \\
& \sum_{i=1}^{\infty} E\left(\left|\phi^{(i)}(\infty, \cdot)\right|^{2} \frac{\left(t-\langle W\rangle_{\infty} \vee t_{0}\right)^{i}}{i !} ; \tau(t, \cdot)=+\infty\right)<\infty .
\end{aligned}
$$

Then $\phi\left(\right.$ resp, $\left.\phi^{(i)}(i=1,2, \cdots)\right)$ admits a power series representation of the following form:

For every $t>0$ :

$$
\phi(\tau(t, \cdot))=\phi(0, \cdot)+\sum_{i=1}^{\infty} \frac{\phi^{(i)}(0, \cdot)}{i !} W_{\tau(t, \cdot)}^{i}
$$

where the convergence of the right-hand side of (15) is to be in $L^{2}-$ norm

(resp. for $i=1,2, \cdots$ :

$$
\phi^{(i)}(\tau(t, \cdot))=\phi^{(i)}(0, \cdot)+\sum_{j=1}^{\infty} \frac{\phi^{(i+j)}(0, \cdot)}{j !} W_{\tau(t, \cdot)}^{j} .
$$

b) Let $\phi=(\phi(t, \cdot))$ be a W-smooth conformal martingale such that

$$
\sum_{i=1}^{\infty} E\left(\left|\phi^{(i)}(\infty, \cdot)\right|^{2} \frac{\left(t-\langle W\rangle_{\infty} \vee t_{0}\right)^{i}}{i !} ; \tau(t, \cdot)=+\infty\right)<\infty,
$$

then for any $t \geq 0$ :

$$
\phi(\tau(t, \cdot))=\phi(0, \cdot)+\sum_{i=1}^{\infty} \frac{\phi^{(i)}(0, \cdot)}{i !} W_{\tau(t, \cdot)}^{i} \quad \text { a.s. } P .
$$

Proof. a) By Lemma $2, \phi=(\phi(\tau(t, \cdot)))$ has the following Taylor expansion:

$$
\phi(\tau(t, \cdot))=\phi(0, \cdot)+\sum_{i=1}^{n} \frac{\phi^{(i)}(0, \cdot)}{i !} W_{\tau(t, \cdot)}^{i}+R_{n+1}(\tau(t, \cdot))
$$

where

$$
R_{n+1}(\tau(t, \cdot))=\phi^{(n+1)} \stackrel{(n+1)}{*} W(\tau(t, \cdot)) .
$$

Let us estimate the $L^{2}$-norm of the rest process $R_{n+1}(\tau(t, \cdot))$ : 


$$
\begin{aligned}
E\left(\left|R_{n+1}(\tau(t, \cdot))\right|^{2}\right) & =E\left(\int_{0}^{\tau(t, \cdot)}\left|\phi^{n+1} \stackrel{*}{*} W\right|^{2}(s, \cdot) d\langle W\rangle_{s}\right) \\
& =E\left(\int_{0}^{t \wedge\langle W\rangle_{\infty}}\left|\phi^{n+1} \stackrel{*}{*} W\right|^{2}(\tau(s, \cdot)) d s\right)
\end{aligned}
$$

By a computation similar to that used in the proof of Proposition 3, a), we get by iteration on the terms $E\left(\left|\phi^{n+1} \stackrel{i}{*} W\right|^{2}\left(\tau\left(t_{i}, \cdot\right)\right)\right)(0 \leq i \leq n)$ :

$$
\begin{aligned}
& E\left(\left|R_{n+1}(\tau(t, \cdot))\right|^{2}\right)=E\left(\int_{0}^{t}\left|\phi^{n+1} * W\right|^{2}\left(\tau\left(t_{n}, \cdot\right)\right) d t_{n} ; \tau(t, \cdot)<+\infty\right) \\
& \quad+E\left(\int_{0}^{\langle W\rangle_{\infty}}\left|\phi^{n+1} \stackrel{*}{*} W\right|^{2}\left(\tau\left(t_{n}, \cdot\right)\right) d t_{n} ; \tau\left(t_{0}, \cdot\right)<\tau(t, \cdot)=+\infty\right) \\
& =E\left(\int_{0}^{t}\left|\phi^{n+1} * W\right|^{2}\left(\tau\left(t_{n}, \cdot\right)\right)\right) d t_{n} \\
& \quad-E\left(\int_{\left\langle W>_{\infty}\right.}^{t}\left|\phi^{n+1} * W\right|^{2}\left(\tau\left(t_{n}, \cdot\right)\right) d t_{n} ; \tau\left(t_{0}, \cdot\right)<\infty, \tau(t, \cdot)=+\infty\right) \\
& =\int_{0}^{t} d t_{n} \int_{0}^{t_{n}} d t_{n-1} \cdots \int_{0}^{t_{1}} E\left(\left|\phi^{n+1}(\tau(s, \cdot))\right|^{2}\right) d s \\
& \quad-\sum_{i=0}^{n}\left(\int_{0}^{t=t_{n+1}} d t_{n} \cdots \int_{0}^{t_{i+2}} d t_{i+1}\right. \\
& \left.\quad \times E\left(\int_{\langle W\rangle_{\infty}}^{t_{i+1}}\left|\phi^{n+1} * W\right|^{2}(\tau(s, \cdot)) d s ; t_{0}<\langle W\rangle_{\infty} \leq t_{i+1}\right)\right) .
\end{aligned}
$$

Hence,

$$
E\left(\left|R_{n+1}(\tau(t, \cdot))\right|^{2}\right) \leq E\left(\left|\phi^{n+1}(\tau(t, \cdot))\right|^{2}\right) \frac{t^{n+1}}{(n+1) !} .
$$

Using the condition (16) we can apply Proposition 3 and we get the following by putting $t=2 t_{0}$ in (15):

$$
\lim _{n \rightarrow \infty} E\left(\left|R_{n+1}(\tau(t, \cdot))\right|^{2}\right) \leq \lim _{n \rightarrow \infty} E\left(\left|\phi^{(n+1)}(\tau(t, \cdot))\right|^{2}\right) \frac{t^{n+1}}{(n+1) !}=0 .
$$

b) By localization the local martingale $\phi=(\phi(t))$ with the sequence of $\left(\mathscr{F}_{\tau(t, .)}\right)$-stopping time $\left(T_{n}\right)$, where

$$
T_{n}=\inf \left\{t>0:|\phi(\tau(t, \cdot))|^{2} \geq n\right\}
$$

we have by the part a):

$$
\phi\left(\tau(t, \cdot) \wedge T_{n}, \cdot\right)=\lim _{m \rightarrow \infty} \sum_{i=0}^{m} \frac{\phi^{(i)}(0, \cdot)}{i !} \cdot W_{\tau(t, \cdot) \wedge T_{n}}^{i} \quad \text { in } L^{2} .
$$

The desired formula (18) comes out by letting $n \rightarrow+\infty$.

q.e.d. 
Remarks. 1) In the case where $\tau(t, \cdot)<+\infty$ a.s., i.e, $\langle W\rangle_{\infty} \geq t$, the condition (14) in Proposition 3 is automatically satisfied and hence (13) becomes:

$$
\begin{aligned}
E\left(|\phi(\tau(t, \cdot))|^{2}\right)= & E\left(\left|\phi\left(\tau\left(t_{0}, \cdot\right)\right)\right|^{2}\right)+\sum_{i=1}^{n} E\left(\left|\phi^{(i)}\left(\tau\left(t_{0}, \cdot\right)\right)\right|^{2}\right) \frac{\left(t-t_{0}\right)^{i}}{i !} \\
& +\int_{0}^{t} d t_{n} \cdots \int_{0}^{t_{1}} E\left(|\phi(\tau(s, \cdot))|^{2}\right) d s .
\end{aligned}
$$

Thus we re-fined here known results of Caroli-Walsh (Lemma 9.13, [4]) and also that of M. Yor (Theorem 1.1, [5]). Furthermore our result answers also an open question raised by Yor in the above mentioned paper (see the remark following Theorem 1.1).

2) Instead of a computation on the expectation if we compute on paths of the increasing process $\left\langle R_{n+1}\right\rangle_{\tau(t, .)}$ then we could obtain the following formula which gives rise to an estimation sharper than (20),

$$
\begin{aligned}
& \left\langle R_{n+1}\right\rangle_{\tau(t, \cdot)}=\int_{0}^{t} d t_{n} \int_{0}^{t_{n}} d t_{n-1} \cdots \int_{0}^{t_{1}}\left\langle\phi^{n+1}\right\rangle_{\tau(s, .)} d s \\
& \quad-\sum_{i=0}^{n}\left|\phi^{n+1} \stackrel{*}{*} W\right|^{2}(\infty, \cdot) \cdot \frac{\left(t-\langle W\rangle_{\infty} \vee t_{0}\right)^{i}}{i !} \cdot \chi_{\left\langle\langle W\rangle_{\infty} \leq t\right)}+M_{\tau(t, \cdot)}
\end{aligned}
$$

where $M=(M(t, \cdot))$ is a process of the form $\sum M_{t}^{i} \cdot t^{i} / i$ ! in which $M^{i}$ are local martingales.

\section{Applications}

1) Approximation of the solution of a complex Ito-diffusion equation.

Let $B=\left(B_{s}\right)$ be the complex Brownian motion, i.e. $B$ is a special conformal martingale of the form $B_{s}=U_{s}+i V_{s}$ where $U=\left(U_{s}\right)$ and $V$ $=\left(V_{s}\right)$ are two independent 1-dimensional Wiener processes for the given probability system $\Sigma=\left(\Omega, \mathscr{F},\left(\mathscr{F}_{t}\right)_{t \geq 0}, P\right)$. Consider the complex Itodiffusion equation:

$$
\phi(t)=\phi(0)+\int_{0}^{t} a^{1}(\cdot, \phi(s)) \cdot d B_{s}
$$

where the process $a^{1}=\left(a^{1}(\cdot, \phi(s))\right)$ is supposed to belong to $L_{\mathrm{loc}}^{2}(B)$.

If the equation (21) has a unique solution $\phi=(\phi(s))$ then we can inspired from our Definition 1 to say that the diffusion $\phi$ admits $a^{1}$ as its $1^{\text {st }}$ derivative. 
Suppose now that the process $a^{1}=\left(a^{1}(\cdot, \phi(s))\right)$ is itself the unique solution of a complex Ito-diffusion equation of the type:

$$
a^{1}(\cdot, \phi(t))=a^{1}(\cdot, \phi(0))+\int_{0}^{t} a^{2}\left(\cdot, a^{1}(\cdot, \phi(s))\right) d B_{s}
$$

then the process $a^{2}=\left(a^{2}\left(\cdot, a^{1}(\cdot, \phi(s))\right)\right)$ is called the $2^{\text {nd }}$ derivative of $\phi$.

By iteration we say that the solution $\phi=(\phi(s))$ of the equation (21) is smooth if it has derivative $\left(a^{i}\right)$ of arbitrary order which can be defined recursively as the unique solutions of the following sequence of diffusion equations:

$$
\begin{gathered}
\phi(t)=\phi(0)+\int_{0}^{t} a^{1}(\cdot, \phi(s)) \cdot d B_{s} \\
\begin{aligned}
a^{1}(\cdot, \phi(t)) & =a^{1}(\cdot, \phi(0))+\int_{0}^{t} a^{2}\left(\cdot, a^{1}(\cdot, \phi(s))\right) \cdot d B_{s} \\
\vdots & \\
a^{i}(\cdot, \phi(t)) & =a^{i}(\cdot, \phi(0))+\int_{0}^{t} a^{i+1}\left(\cdot, a^{i}(\cdot, s)\right) \cdot d B_{s} .
\end{aligned}
\end{gathered}
$$

It turns out that if the solution $\phi=(\phi(s))$ is smooth then it is also $B$ smooth in the sense of Definition 1 and furthermore $\phi^{(i)}=a^{i}(i=1,2, \cdots)$ and $\langle B\rangle_{t}=2 t$. Hence by Theorem $4, \phi$ admits the following power series representation:

$$
\phi(t)=\phi(0)+\sum_{i=0}^{\infty} \frac{a^{(i)}(0)}{i !} \cdot B_{t}^{i} \quad \text { a.s. } P .,
$$

where $\left(a^{(i)}(0)\right)$ is a sequence of $\mathscr{F}_{0}$-measurable functions such that $a^{(1)}(0)=a^{1}(0, \phi(0))$ and $a^{(i)}(0)=a^{i}\left(0, a^{i-1}(0)\right)(i=2,3, \cdots)$.

On the one hand we can approximate in the $L^{2}$-norm the solution $\phi=(\phi(t))$ of (21) by its Taylor expansion $\sum_{i=0}^{n}\left(a^{(i)}(0) / i !\right) \cdot B_{t}^{i}$ and on the other hand the rate of convergence for the approximation procedure could be controlled by the following inequality:

$$
\lim _{n \rightarrow \infty} E\left(R_{n+1}(t)\right) \leq \lim _{n \rightarrow \infty} E\left(\frac{\left|a^{n+1}(t)\right|^{2}}{(n+1) !}\right) \cdot(2 t)^{n+1},
$$

in which the limit in the right hand-side should be zero by (15).

2) Power series expansion of finely holomorphic functions.

Let $U \subset C$ be a finely open and finely connected subset of the complex plane $C$, i.e., $U$ is a fine domain. A complex function $f: U \rightarrow C$ is said 
to be finely holomorphic (see [1]) if for any $x \in U$ there is a compact fine neighbourhood $K_{x} \subset U$ of $x$ such that $\left(f \mid K_{x}\right) \in R\left(K_{x}\right)$. Recall that $R\left(K_{x}\right)$ is the algebra of functions whose elements can be uniformly approximated by a sequence of functions holomorphic in the neighbourhoods of $K_{x}$. Following [3] we will denote by $\mathscr{H}_{\mathrm{f}}(U)$ the algebra of finely holomorphic functions in $U$. In our previous works (see [3]) we showed that there is a fine complex differential $\partial_{U}: \mathscr{H}_{\uparrow}(U) \rightarrow \mathscr{H}_{\uparrow}(U)$, such that for any $f \in \mathscr{H}_{\mathrm{f}}(U)$ and for any $x \in U$ there is a compact fine neighbourhood $K_{x}$ of $x$ :

$$
f\left(B_{T \wedge t}\right)=f\left(B_{0}\right)+\int_{0}^{t} \partial_{U} f\left(B_{T \wedge s}\right) \cdot d B_{T \wedge s} \quad \text { a.s. } P^{x}\left(x \in K^{f}\right),
$$

where $K^{f}$ is the fine interior of $K_{x}$ and where $T$ is the exit-time of $K^{f}$.

Since $\partial_{U}^{i} f \equiv \partial_{U}\left(\partial_{U}^{i-1} f\right) \in \mathscr{H}_{f}(U)(i=12,3, \cdots)$ it follows from (24) that the process $\phi=\left(f\left(B_{T \wedge t}\right)\right)$ is smooth with respect to $W=\left(B_{T \wedge t}\right)$ and its $i^{\text {th }}$ derivative is equal to $\left(\partial_{U}^{i} f\left(B_{T \wedge t}\right)\right)$. Since $P^{x}(T>0)=1$ for any $x \in K^{f}$ and since $\left\langle B_{T \wedge}\right\rangle_{t}=T \wedge t$, we have $\tau(t, \omega)=T(\omega) \wedge t$. By a known result on bounded derivation of the algebra $R\left(K_{x}\right)$ (see [6]) there is a constant $c$ such that $\left|\partial_{U}^{i} f(y)\right| \leq c \cdot\|f\|_{K}$ for every $f \in \mathscr{H}_{f}(U)$ and $y \in K^{f}(i=1,2, \cdots)$, consequently the condition (16) of the Theorem 4 is satisfied for $f\left(B_{T \wedge t}\right)$. The processes $f\left(B_{T \wedge t}\right)$ and $\partial_{U}^{i} f\left(B_{T \wedge t}\right)(i=1,2, \cdots)$ admit power series representations, thus we recover some results of [3].

3) Singularities of smooth conformal martingales.

Let $\phi=\{\phi(t) ; t>0\}$ be a continuous complex process adapted to $\left(\mathscr{F}_{t}\right)_{t \geq 0}$ with parameter set $] 0,+\infty[. \quad \phi$ is said to be smooth with respect to $W \in \mathscr{C}$ off the time " $t=0$ " provided that for every real $t_{0}>0$ the shifted process $\phi\left(\theta_{t_{0}}\right) \equiv\left\{\phi\left(t+t_{0}\right)\right\}_{t \geq 0}$ is smooth with respect to the process $W\left(\theta_{t_{0}}\right) \equiv$ $\left\{W\left(t+t_{0}\right)\right\}_{t \geq 0}$ which, by hypotheses, is conformal for the probability system $\sum_{t_{0}} \equiv\left(\Omega, \mathscr{F},\left(\mathscr{F}_{t+t_{0}}\right)_{t \geq 0}, P\right)$. A process $\phi \in \mathscr{M}_{c}$ is said to have an isolated zero at time " $t=0$ " provided that

$$
P(\phi(0)=0)=1 \text { and } P(\phi(t)=0)=0 \text { for any } t>0 .
$$

The proof of the following theorem is similar to that of Theorem 7 in [3].

Theorem 5. Let $W \in \mathscr{C}$ be a fixed conformal martingale with only isolated zero at " $t=0$ " and let $\phi=\{\phi(t)\}_{t>0}$ be a smooth process off the time " $t=0$ " with respect to $W$. Then one of the following three cases must occur: 
a) The process $\phi$ can be extended to a smooth process with respect to $W$ on the whole interval $[0,+\infty[$. In this case $\phi$ is said to have a $W$ removable singularity at the time " $t=0$ ".

b) There are $m$ complex numbers $c_{1}, \cdots, c_{m}$ where $m$ is a positive integer and $c_{m} \neq 0$ such that the process,

$$
\phi-\sum_{i=1}^{m} c_{i} W^{-i}
$$

can be extended to be W-smooth on $[0,+\infty[$. In this case $\phi$ is said to have a $W$-pole of order $m$ at time " $t=0$ ".

c) for each $\delta>0$ the set,

$$
\{(\phi(\omega, t)) ;(\omega, t) \in \mathbb{0}, \delta \llbracket\}
$$

is dense in the complex plane $C$. In this case $\phi$ is said to have a $W$ essential singularity at time " $t=0$ ".

Remarks. 1) Note that since we have only to study the process near the time " $t=0$ " thus by localization we can suppose without loss of generalities that the process satisfies the condition (16) of Theorem 4.

2) Our result generalizes that of Walsh in [7] in the sense that if the conformal process is smooth of the time " $t=0$ " then we can classify its pole at " $t=0 "$.

Acknowledgement. The author would like to thank the referee for pointing to him an error in the proof of Proposition 3 and for giving him many interesting comments.

\section{REFERENCES}

[1] B. Fuglede, Fine topology and finely holomorphic functions, Proc. 18th. Scandinavian Cong. of Mathematicians, Birkhäuser, (1981).

[2] R. K. Getoor and M. J. Sharpe, Conformal martingales, Invent. Math., 16 (1972), 271-308.

[ 3 ] Nguyen-Xuan-Loc, Singularities of locally analytic processes, Proc. Symp. on Potential Theory, Lect. Notes in Math., No. 787, Springer-Verlag (1980), 267-288.

[4] R. Caroli and J. B. Walsh, Stochastic integrals in the plane, Acta. Math., 134 (1975), 111-183.

[5] M. Yor, Etude de certains processus (stochastiqument) differentiables ou holomorphes, Ann. Inst. Henri Poincaré, XIII, No. 1 (1977), 1-25.

[6] Debiard et B. Gaveau, Potentiel fin et algebre de fonctions analytiques, J. Funct. Anal., 16 (1974), 289-304. 
[ 7 ] J. B. Walsh, A property of conformal martingales, Seminaire de Probabilities 11, Lect. Notes in Math., No. 581, Springer-Verlag (1975/76), 490-492.

Department of Mathematical Statistics

Institute of Computer Science and Cibernetics

Lieu Giai Ba Dinh

Ha Noi R. S. Viet Nam 